\title{
Mechanistic investigation and free energies of the reactive adsorption of ethanol at the
} alumina/water interface

Paul Clabaut, ${ }^{\dagger}$ Jérôme Rey, ${ }^{\dagger}$ Romain Réocreux, ${ }^{\ddagger}$ Stephan N. Steinmann, ${ }^{+}$and Carine Michel ${ }^{*, \dagger}$

+Univ Lyon, ENS de Lyon, CNRS UMR 5182, Laboratoire de Chimie, F69342, Lyon, France $\ddagger$ Thomas Young Centre and Department of Chemical Engineering, University College London, Roberts Building, Torrington Place, London WC1E 7JE, United Kingdom

E-mail: carine.michel@ens-lyon.fr

\begin{abstract}
Controlling the adsorption/desorption of molecules at the solid/water interface is central to a diversity of fields from catalysis to batteries. Preventing the desorption of alcohol at the $\gamma-\mathrm{Al}_{2} \mathrm{O}_{3} /$ water interface is key to increase the stability of this catalyst support to perform reactions in water. Taking ethanol as a typical example, we investigate here the mechanism of desorption of two adsorption modes, namely chemisorbed ethanol and ethoxy, from the interface to the bulk water using three DFT-based simulations. Our 3D well-tempered metadynamics simulations include a bias in solvation, which triggers possible proton transfers with water. They evidence that solvation needs to be increased prior to desorption in both cases. Comparison with static approaches and thermodynamic integration simulations unambiguously identifies ethoxy as the more stable adsorption
\end{abstract}


mode. It is more stable by at least $40 \mathrm{~kJ} \cdot \mathrm{mol}^{-1}$ when considering adsorption at the gas/solid interface. And the presence of liquid water yields to a desorption barriers ranging from 89 $\mathrm{kJ} \cdot \mathrm{mol}^{-1}$ (thermodynamic integration) to $149 \mathrm{~kJ} \cdot \mathrm{mol}^{-1}$ (well-tempered metadynamics). The observed difference between the two biased ab initio molecular dynamics methods can be ascribed to the intrinsic difficulty of sampling the desorbed state vs. the adsorbed state.

\section{Introduction}

Understanding the adsorption/desorption at the solid/water interface is key in tribology, ${ }^{1}$ heterogeneous catalysis, ${ }^{2}$ electrochemistry, ${ }^{3,4}$ chromatography, etc. The small volume of the interface compared with the volume of both the bulk liquid water and the bulk solid challenges experimental investigations. ${ }^{5-7}$ Available information about water structuration at these interfaces are therefore limited to very thin water layers under high-vacuum conditions (obtained via spectroscopy ${ }^{8,9}$ or microscopy ${ }^{7}$ ) or under applied electric potentials (deduced from electrochemical measurement). ${ }^{10}$ Moreover, water may dissociate when chemisorbed on metals, ${ }^{11}$ oxides, ${ }^{12}$ and other materials (fluorite for instance ${ }^{13}$ ), and its liquid structure is believed to depend on the nature and/or the morphology of the surface. ${ }^{5,7}$ Atomistic modelling appears as a complementary tool of choice to gain detailed understanding beyond spectroscopic signatures and it has been extensively applied to study the structuration of interfacial water, ${ }^{7}$ but also the adsorption of molecules or ions at the solid/water interface. ${ }^{14,15}$

Modelling a solid/water interface requires an extensive sampling of the phase space of the liquid water. ${ }^{16}$ In most cases, the dynamic of water is slowed down at the interface by several orders of magnitude, making the sampling of such a system challenging. ${ }^{17}$ Nonreactive adsorptions can be treated with classic molecular mechanics. ${ }^{14,18,19}$ Combining this classic description with an ab initio description of the surface/adsorbate interaction im- 
proves the energetics of adsorption. ${ }^{20-24}$ For instance, using our recent MMSolv approach, the adsorption energy at the water/Pt interface of phenol and benzene was predicted semi-quantitatively. ${ }^{25}$ This hybrid scheme highlighted the importance of the desolvation of the surface in limiting the adsorption at the interface.

Reactive adsorptions are typically more challenging to investigate. During an adsorption process, changes in solvation may be combined with reactions that involve the water solvent, the adsorbate and/or the surface, and those reactions cannot be easily disentangled from the desorption/adsorption process. Typically, proton-shuffling between the water solvent, the adsorbate (e.g. an alcohol, a phosphate) and the surface (e.g. an oxide) are likely to accompany the adsorption of protic molecules. Therefore, accounting for proton transfers can be crucial when studying reactive adsorptions. Reactive force fields ${ }^{26-28}$ or semi-empirical methods such as DFT-B ${ }^{29,30}$ appear as a good strategies since they offer the possibility to investigate and sample reactions at a reasonable cost. More recently, force field based on machine learning techniques, such as neural networks, were also successfully developed to investigate solid/liquid interfaces. ${ }^{31-33}$ However these methods fail at handling increased numbers of atom types. More generally, using empirical methods to study reactive adsorption requires the development of $a d$ hoc parameters for each system, which represents a severe limitation that can be overcome moving to Density Functional Theory (DFT). Investigating reactions at the solid/water interface using DFT can be performed by biasing ab initio molecular dynamics along selected collective variables using rare event methods such as metadynamics, ${ }^{34-37}$ umbrella sampling ${ }^{38}$ or thermodynamic integration. ${ }^{39,40}$ Combining different theoretical methods was shown to provide a better understanding of the mechanisms in complex environments and in presence of water. ${ }^{41,42}$ Using ab initio metadynamics, we have recently investigated the mechanism of hydrolysis of $\gamma-\mathrm{Al}_{2} \mathrm{O}_{3}$, an important support in heterogenous catalysis, when it is immersed in water. We have shed light on the role of the adsorption of some polyols at the $\gamma-\mathrm{Al}_{2} \mathrm{O}_{3}$ /water interface in preventing hydrolysis. ${ }^{43}$ Although there is evidence that alcohols chemisorb 
on $\gamma-\mathrm{Al}_{2} \mathrm{O}_{3},{ }^{44,45}$ the details of the adsorption sites and energetics are yet to be elucidated. This lack of understanding of the adsorption process of alcohols at the $\gamma-\mathrm{Al}_{2} \mathrm{O}_{3} /$ water interface makes the quest of better protecting additives empirical.

Herein, we investigate the adsorption of ethanol, taken as a typical alcohol, at the $\gamma-\mathrm{Al}_{2} \mathrm{O}_{3} /$ water interface combining three simulation methods to unravel the mechanism of adsorption and the relative stability of two adsorption modes. We first explore the desorption mechanism of ethanol using ab initio metadynamics to build free energy surfaces in three dimensions, including a solvation variable. Then, focusing on the key intermediates, we estimate reaction free energies using a static approach combined with different models accounting for solvation. Last, we refine the energetics of the different processes using ab initio thermodynamic integration.

\section{Computational details}

\subsection{Models of $\gamma-\mathrm{Al}_{2} \mathrm{O}_{3} /$ water interface}

Our reference model $S_{r e f}$ of the $\gamma-\mathrm{Al}_{2} \mathrm{O}_{3}(110)$ surface in contact with liquid water was taken as the last frame of the ab initio molecular dynamics (AIMD) trajectory we published previously. ${ }^{17}$ Briefly, a periodic slab of the $\gamma-\mathrm{Al}_{2} \mathrm{O}_{3}(110)$ surface of $10 \AA$ thickness was cleaved using the bulk model of $\gamma-\mathrm{Al}_{2} \mathrm{O}_{3}$ proposed by Krokidis et al. ${ }^{46}$ Then, we hydrated the top surface ${ }^{47}$ of a $\mathrm{p}(2 \times 2)$ cell and took into account the surface reconstruction that can happen in contact with water as evidenced by Wischert et al. ${ }^{48}$ We put the obtained hydrated surface in contact with a slab of liquid water of $20 \AA$. The periodic images perpendicular to the interface were separated by a void of $10 \AA$. Proton transfers were observed at the interface during the trajectory of $74 \mathrm{ps}$ (including equilibration). The surface state of the resulting $\mathrm{p}(2 \times 2)$ cell of $S_{\text {ref }}$ is represented schematically in Figure 1(a). The original primitive cell (highlighted with a square) exposes two octahedral aluminium atoms ((1) and (2) following Copeland's denomination $\left.{ }^{44}\right)$ and two tetrahedral aluminium 
atoms $(\alpha$ and $\beta$ ) at the surface. In the $\mathrm{p}(2 \times 2)$ cell, 20 water molecules are chemisorbed, completing the aluminium coordination up to 4 or 6 depending on the sites. 13 of these water molecules are dissociated, generating 26 hydroxyl groups at the surface. In this work, we have identified and considered an other surface state with one more dissociated water molecule. It is referred to as $S_{d i s s}$ and shown in Figure 1(b).
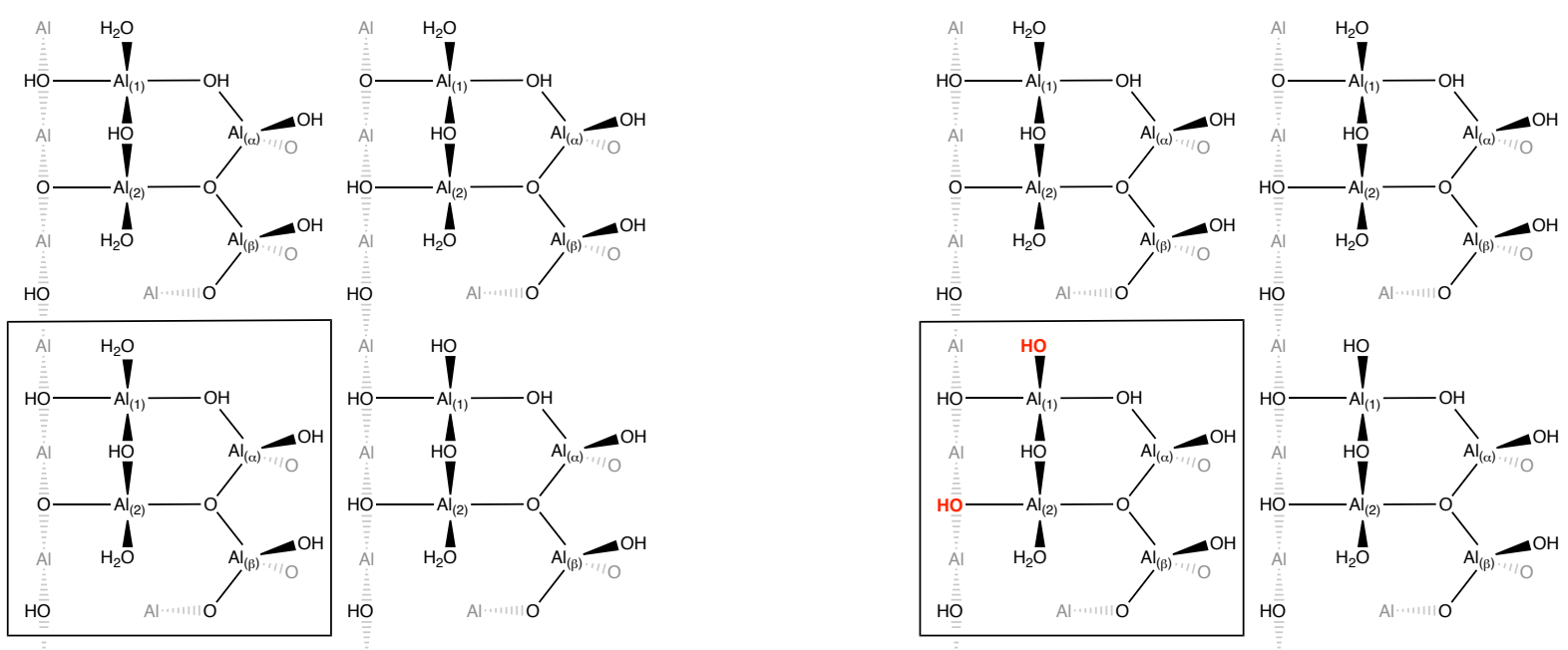

(a) $S_{\text {ref }}$

(b) $S_{\text {diss }}$

Figure 1: Schematic representation of the $\gamma-\mathrm{Al}_{2} \mathrm{O}_{3}(110) /$ water interface in two surface states: (a) $S_{r e f}$ and (b) $S_{\text {diss }}$. The atoms involved in the reshuffling of hydrogen are showed in red. 


\subsection{Desorption/Adsorption of ethanol at the $\gamma-\mathrm{Al}_{2} \mathrm{O}_{3} /$ water interface}

We studied the desorption of ethanol from the $\gamma-\mathrm{Al}_{2} \mathrm{O}_{3}(110) /$ water interface to the bulk water considering two configurations of ethanol that differ by the number of $\mathrm{Al}-\mathrm{O}_{\mathrm{EtOH}}$ bonds (see Fig 2). This number of bonds is called multiplicity later. In analogy with coordination chemistry, when the multiplicity is 1 or 2 , the configuration is named $\mu_{1}$ and $\mu_{2}$ respectively. By extension, $\mu_{0}$ refers to ethanol desorbed in the bulk of the water slab. In the configuration $\mu_{1} @ S_{r e f}$, the ethanol molecule interacts through its oxygen with one aluminium atom of $S_{r e f}$, namely the octahedral $\mathrm{Al}_{(2)}$, substituting a non-dissociated water molecule in $S_{r e f}\left(O_{\mu_{1}}\right.$ in Fig $2 \mathrm{a}$ and $\left.\mathrm{b}\right)$. In the configuration $\mu_{2} @ S_{r e f}$, the ethanol is dissociated, bridging two aluminium atoms $\left(\mathrm{Al}_{(1)}\right.$ and $\left.\mathrm{Al}_{(2)}\right)$. It replaces the hydroxyl group that bridges those two aluminium atoms in $S_{r e f}\left(O_{\mu_{2}}\right.$ in Fig 2a and b).

a)
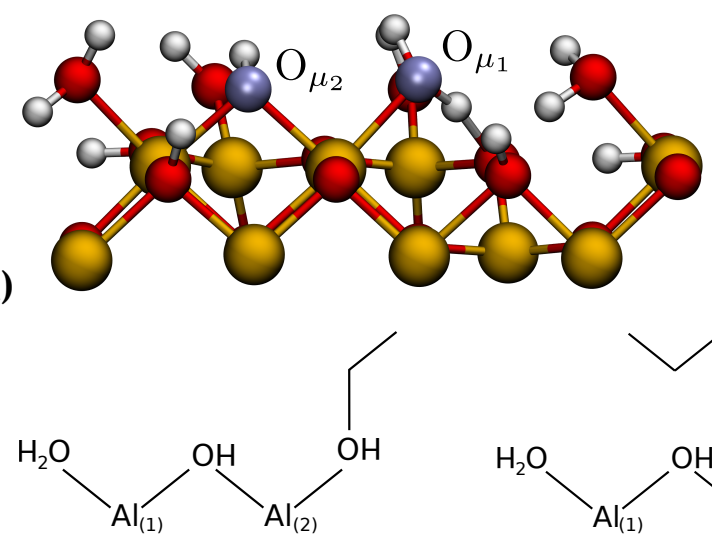

c)

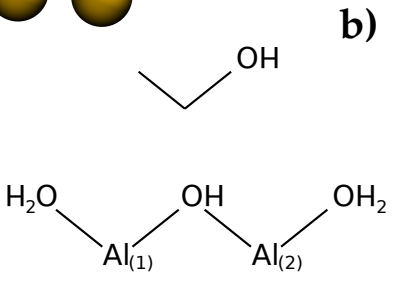

$\mu_{0}$
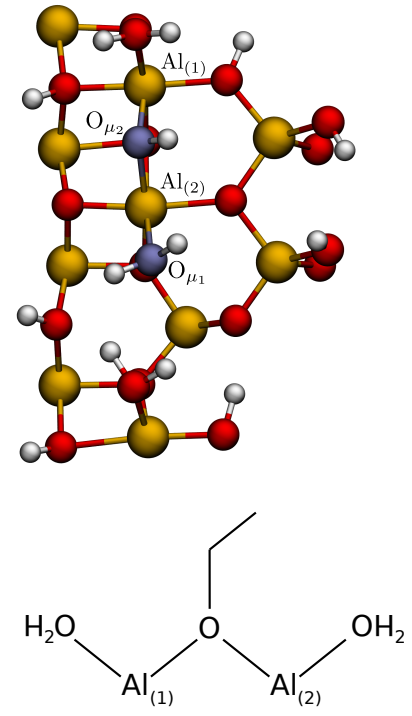

$\mu_{2}$

Figure 2: Hydrated $\mathrm{Al}_{2} \mathrm{O}_{3}(110)$ surface used as the reference surface $S_{r e f}$ : a) side and and $\mathbf{b}$ ) top view. The light blue oxygen atoms labelled $\mathrm{O}_{\mu_{1}}$ and $\mathrm{O}_{\mu_{2}}$ are the two atoms that are substituted to build the $\mu_{1}$ and $\mu_{2}$ adsorption mode respectively. c) Schematic representation of the adsorption of a free ethanol molecule (referred to as $\mu_{0}$ ) to the $\mu_{1}$ and $\mu_{2}$ adsorption mode. 


\subsection{Biased AIMD}

\subsection{General parameters}

Energies and forces were computed using the CP2K-Quickstep ${ }^{49-52}$ implementation of DFT with the Gaussian Plane Wave (GPW) approach, combining a MOLOPT double- $\zeta$ basis set (DZVP) and an auxiliary plane waves basis set with a 400 Ry cutoff for valence density. Goedecker-Teter-Hutter (GTH) pseudo-potentials ${ }^{53-55}$ were used for core electrons. The Perdew-Burke-Ernzerhof (PBE) functional ${ }^{56}$ was supplemented by Grimme D3 correction ${ }^{57}$ to describe electron interactions. The Self-Consistent Field convergence criterion was set to $5 \cdot 10^{-6}$ Hartree.

Dynamic samplings were performed in the NVT thermodynamic ensemble, keeping the temperature held constant at $330 \mathrm{~K}$ via the Canonical Sampling through Velocity Rescaling (CSVR) thermostat. ${ }^{58}$ The two bottom-most layers of alumina were held frozen during dynamics. An integration time step of $0.5 \mathrm{fs}$ was chosen for runs associated with thermodynamic integration, while $1 \mathrm{fs}$ was used for metadynamics runs, associated with an increase tripled atomic weight for hydrogen in order to improve the efficiency of the sampling. Since position-dependent observables are independent of the atomic masses once the convergence is achieved, this choice does not influence the resulting energies. ${ }^{59}$

\subsubsection{Well-tempered metadynamics}

We performed two well-tempered metadynamics ${ }^{60}$ simulations to investigate the desorption of ethanol from the $\gamma-\mathrm{Al}_{2} \mathrm{O}_{3}(110) /$ water interface to the bulk water, one starting from $\mu_{1} @ S_{r e f}$ and the other one starting from $\mu_{2} @ S_{r e f}$. The bias potential was constructed by adding gaussian hills (initial height of $3.3 \mathrm{~kJ} \cdot \mathrm{mol}^{-1}$, bias factor of 100 and temperature of $300 \mathrm{~K}$ ) every $10 \mathrm{fs}$ in the space described by three collective variables (CVs). The set of collective variables were chosen to be as close as possible but were adapted to the specificities of those two adsorption modes: 
- The height $\left(C V_{\text {height }}\right)$ was defined as the absolute coordinate of the ethanol's oxygen in the out-of-plane direction. The width of the Gaussian hills along this CV was set to $0.04 \AA$.

- The multiplicity $\left(C V_{\text {multi }}\right)$ was defined as the coordination between the ethanol's oxygen and the initial adsorption site of alumina: $\mathrm{Al}_{(2)}$ for the metadynamics starting from $\mu_{1} @ S_{\text {ref }}$ (see Fig. 2); and $\mathrm{Al}_{(1)}$ and $\mathrm{Al}_{(2)}$ for the metadynamics starting from $\mu_{2} @ S_{r e f}$ (see Fig. 2). The width of the Gaussian hills along this CV was set to 0.04 .

- The solvation $\left(C V_{\text {solv }}\right)$ was designed to account for the changes in solvation of ethanol and alumina upon desorption. These changes were described through the number of hydrogen bonds between the water solvent and the hydroxyl group of ethanol and the number of $\mathrm{Al}-\mathrm{O}_{\text {water }}$ bonds. $C V_{\text {solv }}$ was defined as follow:

$$
C V_{\text {solv }}=C\left(O_{\text {ethanol }} ; H_{\text {water }} \& H_{\text {ethanol }}\right)+C\left(O_{\text {water }} \& O_{\text {ethanol }} ; H_{\text {ethanol }}\right)+C\left(A l_{n} ; O_{\text {water }}\right)
$$

where $C(A, B)$ stands for the coordination number between two groups of atoms ( $A$ and $B$ ). $O_{\text {ethanol }}$ corresponds to the oxygen of ethanol. $H_{\text {water }}$ and $O_{\text {water }}$ include all hydrogen atoms and all oxygen atoms respectively that originate from water molecules (free, adsorbed, or dissociated). $H_{\text {ethanol }}$ stands for the hydrogen atom of the hydroxyl group of ethanol and is therefore defined only for the adsorption mode $\mu_{1}$. $A l_{n}$ stands for aluminium atoms bonded to ethanol, i.e. $\mathrm{Al}_{(2)}$ when starting with the $\mu_{1} @ S_{r e f}$ adsorption mode, and $\mathrm{Al}_{(1)}$ and $\mathrm{Al}_{(2)}$ when starting with the $\mu_{2} @ S_{r e f}$ adsorption mode. Combining $H_{\text {water }}$ with $H_{\text {ethanol }}$ and $O_{\text {water }}$ with $O_{\text {ethanol }}$ allows to avoid the change of the $\mathrm{CV}$ upon proton exchange between the hydroxyl group of ethanol and water.

The coordination numbers between two groups of atoms A and B used in the above definition were defined following the PLUMED implementation: ${ }^{61}$ 


$$
C(A ; B)=\sum_{i \in A} \sum_{j \in B} s_{i j}
$$

with

$$
s_{i j}=\frac{1-\left(\frac{r_{i j}-d_{0}}{r_{0}}\right)^{n}}{1-\left(\frac{r_{i j}-d_{0}}{r_{0}}\right)^{n}}
$$

with $r_{i j}$ the distance between the atoms $i$ and $j$, and the $d_{0}$ and $r_{0}$ two cut-off distances chosen as presented in Table 1.

Table 1: Numerical parameters used to define the coordination numbers between atoms.

\begin{tabular}{lcccc}
\hline type & $\mathrm{d}_{0}(\AA)$ & $\mathrm{r}_{0}(\AA)$ & $\mathrm{n}$ & $\mathrm{m}$ \\
\hline $\mathrm{O} ; \mathrm{H}$ & 1.9 & 0.4 & 4 & 10 \\
$\mathrm{Al} ; \mathrm{O}$ & 2.0 & 0.8 & 4 & 10 \\
\hline
\end{tabular}

\subsubsection{Thermodynamic integration}

The first 5 ps of each run were considered as equilibration and thus discarded for analysis, leaving production trajectories of at least 10 ps each. At $C V_{\text {height }}>14 \AA$, the configurations of the TI run starting from $\mu_{1} @ S_{r e f}$ and from $\mu_{2} @ S_{r e f}$ correspond both to a free ethanol molecule in liquid water, and only the free energy profile corresponding to the TI run starting from $\mu_{2} @ S_{\text {ref }}$ was used. Similarly, at $C V_{\text {height }}>12.5 \AA$, the structures of the TI run starting from $\mu_{2} @ S_{\text {diss }}$ are similar to those of the TI run starting from $\mu_{2} @ S_{r e f}$ as the surface has undergone spontaneous reconstruction from $S_{d i s s}$ into $S_{r e f}$. Therefore, the free energy profile starting from $\mu_{2} @ S_{\text {diss }}$ was not extended beyond $\mathrm{CV}_{\text {height }}=13 \AA$.

\subsubsection{Computing free energy differences between macro-states}

To avoid the arbitrary choice of a single micro-state to represent the macroscopic system, free energy differences between macro-states were computed following the method of Ciacchi et al. ${ }^{37}$ The portion of the space corresponding to a macro-state is defined by 
boundaries $\left(C V_{i, \max }\right.$ and $\left.C V_{i, \min }\right)$ along each $C V_{i}$. The free energy difference between two macro-states 1 and 2 of respective populations $p_{1}$ and $p_{2}$ is given by the formula:

$$
\Delta F=-R T \cdot \ln \left(\frac{p_{1}}{p_{2}}\right)
$$

where $p_{1}$ and $p_{2}$ are defined as follows:

$p\left(C V_{i, \text { min }}, C V_{i, \text { max }}, i=1 . . N_{C V}\right)=\frac{1}{\prod_{C V_{i}}\left(C V_{i, \text { max }}-C V_{i, \text { min }}\right)} \int_{C V_{1, \text { min }}}^{C V_{1, \max }} \cdots \int_{C V_{N, \text { min }}}^{C V_{N, \max }} \exp \left(-\frac{F\left(x_{C V}^{N}\right)}{R T}\right) d x_{C V}^{N}$

with $x_{C V}^{N}$ the coordinate of the system in the $\mathrm{N}$-space defined by the CVs.

Similarly, representation of 3D free energy surfaces as 2D surfaces in section 3.1 were produced using the equation 5 to compute the population $p\left(C V_{1}, C V_{2}\right)$ integrating out the variable $C V_{3}$ and then deriving $F\left(C V_{1}, C V_{2}\right)=-R T \cdot \ln \left(p\left(C V_{1}, C V_{2}\right)\right)$ within a constant.

\subsection{General parameters for static DFT computations with VASP}

For static DFT computations, electronic energies were computed using the plane-wave VASP 5.4.1 code. ${ }^{62,63}$ The PBE functional was used to describe electron interactions, ${ }^{56,64}$ supplemented by the $\mathrm{dDsC}$ dispersion correction. ${ }^{65,66}$ The electron-ion interactions were described by the PAW formalism. ${ }^{67,68}$ The plane-wave energy cutoff was set to $400 \mathrm{eV}$. This choice was made to be compatible with our implementation of the MMSolv method (see below).

For implicit solvent computations, the Polarisable Continuum Model (PCM) implemented in the VASPsol module ${ }^{69}$ was used. The default settings for cavitation energy were used.

The entropic difference associated with ethanol adsorption was estimated neglecting the vibrational contributions. Hence, the terms corresponding to the adsorbed states EtOH@S and $\mathrm{H}_{2} \mathrm{O} @ S$ cancel out, and the entropic contribution can be written: 


$$
\Delta_{a d s} S=-S_{a q}(\mathrm{EtOH})+S_{a q}\left(\mathrm{H}_{2} \mathrm{O}\right)
$$

To take into account the effect of the bulk solvent on the translational and rotational entropy, we took the experimental value of entropy for a water molecule solvated in water $\left(S_{a q}\left(\mathrm{H}_{2} \mathrm{O}\right)\right)$ and estimated $\left(S_{a q, r o t}(\mathrm{EtOH})\right.$ and $\left.S_{a q, \text { trans }}(\mathrm{EtOH})\right)$ by scaling the perfect gas and rigid rotator entropy of ethanol using the empiric relation established by Wertz and co-workers: ${ }^{70}$

$$
S_{a q}(E t O H)=0.54 \cdot S_{g a s}(E t O H)+2.76 \cdot 10^{-2}\left(\mathrm{~kJ} \cdot \mathrm{mol}^{-1} \cdot \mathrm{K}^{-1}\right)
$$

\subsection{Molecular mechanics computations with AMBER}

The MMSolv computations were conducted using the method and workflow described in our previous work for the evaluation of the adsorption free energy of benzene or phenol on a Pt(111) surface. ${ }^{25}$ The $\gamma$-alumina slab is frozen. Lennard-Jones parameters for $\gamma$-alumina atoms are taken from the CLAYFF forcefield. ${ }^{71}$ A gaussian attractive potential was added between the oxygens of water molecules and surface aluminum atoms to improve the description of the chemisorption. ${ }^{72}$ Chemisorbed water molecules (dissociated or not) were also frozen. Their Lennard-Jones parameters were taken from the UFF forcefield, ${ }^{73}$ and their partial charges were extracted from DFT static computations of the hydrated slab, following our previously described MMSolv method. ${ }^{25}$

\section{Results and discussion}

To investigate the mechanism of adsorption/desorption of ethanol at the $\gamma-\mathrm{Al}_{2} \mathrm{O}_{3}(110) /$ water interface, we compared two configurations: (i) in $\mu_{1} @ S_{r e f}$, ethanol is chemisorbed interacting with a single aluminium atom of the reference surface $S_{r e f}$; (ii) in $\mu_{2} @ S_{r e f}$, ethanol is adsorbed dissociatively and bridges two aluminium atoms of the reference surface $S_{r e f}$. 
More details are provided in section 2.1 and 2.2. The corresponding structures are sketched in Figure 1 and 2. The mechanism was investigated using ab initio metadynamics. Then, using this mechanistic information, the energetics was refined through static models and ab initio thermodynamic integration (TI).

\subsection{Investigating the mechanism of desorption using ab initio metady- namics}

To drive the desorption of ethanol from the $\gamma-\mathrm{Al}_{2} \mathrm{O}_{3}(110) /$ water interface to the bulk water, the height of ethanol is a natural collective variable $\left(C V_{\text {height }}\right)$. Including also the number of bonds of ethanol to the alumina surface (multiplicity, $C V_{\text {multi }}$ ) is also necessary to distinguish between the two chemisorbed macro-states $\left(\mu_{1}\right.$ and $\left.\mu_{2}\right)$ and the desorbed macro-state $\left(\mu_{0}\right)$. Last, the change in solvation happens to be critical and is accounted for using $C V_{\text {solv }}$. This collective variable drives the hydration of ethanol but also the hydration of the vacant coordination site(s) at the alumina surface created during the ethanol desorption. Its definition slightly differs in the two simulations to adapt to the ethoxy $\left(\mu_{2} @ S_{r e f}\right)$ vs. ethanol $\left(\mu_{1} @ S_{r e f}\right)$ starting-point. More details can be found in 2.4.1

Starting from the chemisorbed ethanol $\mu_{1} @ S_{r e f}$, we obtained the free energy surface $\mathrm{FES}_{1}$ represented in Figure 3 through two bi-dimensional representations. The phase-space corresponding to the macro-state $\mu_{1}\left(\mathrm{CV}_{\text {multi }}<0.5\right.$, shown in blue in Figure 3$)$ is small in volume: $\mathrm{CV}_{\text {height }}<12 \AA$ and $\mathrm{CV}_{\text {solv }}=3$ or 4 . In contrast, the portion associated to the macro-state $\mu_{0}\left(\mathrm{CV}_{\text {multi }}>0.5\right.$, shown in green in Figure 3$)$ is large, covering several wells corresponding to variations in solvation from 3 to 7 . As a consequence, only a qualitative estimation of desorption free energy could be obtained since, once the ethanol molecule leaves the interfacial zone, it starts exploring all of the possible solvated states and has difficulty coming back to the original binding site. From the $\mathrm{FES}_{1}$, a desorption energy of $\Delta F_{\text {desorption }}\left(\mu_{1} @ S_{r e f} \rightarrow \mu_{0} @ S_{r e f}\right)=-9 \mathrm{~kJ} \cdot \mathrm{mol}^{-1}$ can be derived, but it is likely underestimated due to the unavoidable lack of sampling of the extended desorbed macro- 
state $\mu_{0} @ S_{r e f}$. The free energy barrier of desorption is likely to be more quantitative $\left(\Delta_{\text {desorption }} F^{\ddagger}\left(\mu_{1}\right)=35 \mathrm{~kJ} \cdot \mathrm{mol}^{-1}\right)$. Improved energetics will be provided through other methods below.

The analysis of the $\mathrm{FES}_{1}$ provides a detailed mechanism of desorption from $\mu_{1} @ S_{r e f}$. The solvation increases upon desorption (from 3 to 8 ). In the chemisorbed sate $\mu_{1} @ S_{r e f}$, the ethanol molecules sits $<2 \AA$ away from to the surface, with one dissociated water molecule co-adsorbed on $\mathrm{Al}_{(2)}$. It features two possible $\mathrm{H}$-bonds with water: one $\mathrm{H}_{\mathrm{EtOH}} \cdots \mathrm{O}_{\text {water }}$ bond and one $\mathrm{O}_{\mathrm{EtOH}} \cdots \mathrm{H}_{\text {water }}$ bond (see Figure S1). Considering the $\mathrm{FES}_{1}$ in Figure 3, the increase in solvation starts prior to the increase in height. Decomposing this solvation collective variable into its components (see Figure S1) shows that the increase in solvation starts with an increase in the number of $\mathrm{O}_{\mathrm{EtOH}} \cdots \mathrm{H}_{\text {water }} \mathrm{H}$-bonds from 1 to 2 and then is followed by an increase of the number of $\mathrm{H}_{\mathrm{EtOH}} \cdots \mathrm{O}_{\text {water }} \mathrm{H}$-bonds and $\mathrm{Al}(1)-\mathrm{O}_{\text {water }}$ bonds. This solvation-shell reorganisation is activated, with energy barriers of approximately $30 \mathrm{~kJ} \cdot \mathrm{mol}^{-1}$ along the solvation coordinate as shown by $\mathrm{FES}_{1}$ (Figure $3 \mathrm{~b}$ )). This is not only important to better understand the desorption mechanism, but it also rationalises the necessity of including the solvation as a collective variable to sample the phase space associated with desorption at solid/liquid interfaces. 
a)

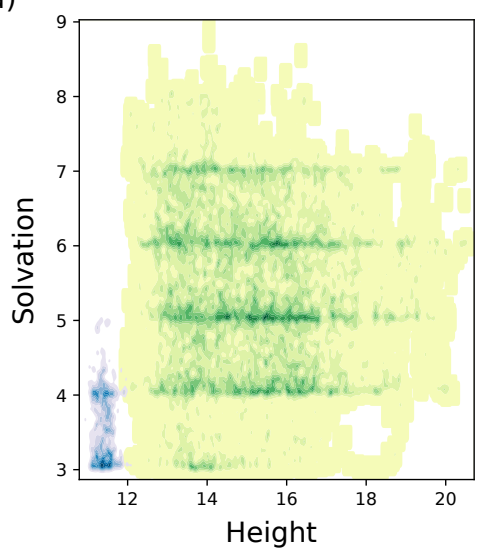

b)

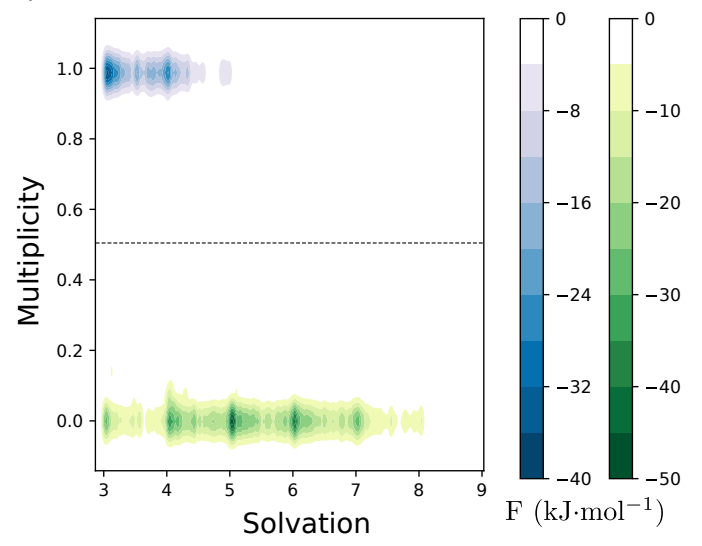

c)
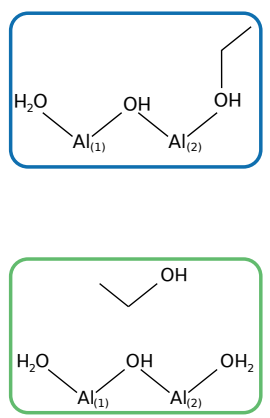

Figure 3: Free energy surface $F E S_{1}$ built using well-tempered metadynamics starting from $\mu_{1} @ S_{r e f}$, with the ethanol chemisorbed at the $\gamma-\mathrm{Al}_{2} \mathrm{O}_{3}(110) /$ water interface. (a) 2Drepresentation of the $3 \mathrm{D} F E S_{1}$ as a function of $\mathrm{CV}_{\text {solv }}$ and $C \mathrm{~V}_{\text {height }}$ (b) 2D-representation of the 3D FES 1 as a function of $C V_{\text {multi }}$ and $C V_{\text {solv }}$. (c) Schemes of a typical arrangement of $\mu_{1} @ S_{r e f}$ (blue) and $\mu_{0} @ S_{r e f}$ (green). In (a) and (b), the $\mu_{1} @ S_{r e f}$ macro-state $\left(\mathrm{CV}_{\text {multi }}>0.5\right.$, chemisorbed ethanol) is shown using a blue scale and the $\mu_{0}$ macro-state $\left(\mathrm{CV}_{\text {multi }}<0.5\right.$, desorbed ethanol) using a green scale. The boundary $\mathrm{CV}_{\text {mult } i}=0.5$ is shown using a grey dotted line. (a) and (b) share the same free energy scales. Details about the reduction in dimension from $3 \mathrm{D}$ to $2 \mathrm{D}$ can be found in section 2.4.3. 
Starting from the chemisorbed ethoxy $\mu_{2} @ S_{r e f}$, well-tempered metadynamics resulted in the free energy surface $\mathrm{FES}_{2}$ represented in Figure 4. $\mathrm{FES}_{2}$ can be divided into three portions: the bridging adsorbed ethoxy macro-state $\mu_{2}$, the mono-dentate macro-state $\mu_{1, b i s}$ where only one Al-O bond still connects the ethanol to the surface, and the desorbed macro-state $\mu_{0}$. Similarly to $\mathrm{FES}_{1}$, the two chemisorbed states $\left(\mu_{2}\right.$ and $\mu_{1, b i s}$ ) correspond to a small volume of the phase space, with a height below $12 \AA$ while the desorbed state $\mu_{0}$ corresponds to a much wider phase space. A total barrier of $\Delta F^{\ddagger}\left(\mu_{2} \rightarrow \mu_{0}\right)=149$ $\mathrm{kJ} \cdot \mathrm{mol}^{-1}$ was found, assorted with a total desorption free energy of $\Delta F=97 \mathrm{~kJ} \cdot \mathrm{mol}^{-1}$. This desorption free energy is here again qualitative due to the lack of sampling of the desorbed state.

Here again the solvation plays a key role and needs to be increased from 2 to 3 prior desorption (see Figure 4). Once ethanol leaves the interface to the bulk water, the alumina surface gets hydrated and the solvation-shell of ethanol builds up, with a solvation spanning a range of 5 to 7 . While the later increase is similar to the one observed for ethanol desorption from $\mu_{1} @ S_{r e f}$, the first increase in solvation from 2 to 3 differs. It happens while the ethanol is still close to the alumina surface, less than $2 \AA$ away from the surface $\left(C V_{\text {height }}<12 \AA\right)$. It corresponds to the protonation of the ethoxy $\mu_{2}$ according to the decomposition of the solvation collective variable into its components (see Figure S2) and to a visual inspection of the trajectory. Importantly, the solvation collective variable triggers proton-reshuffling at the interface that results here into a change in surface state from $S_{r e f}$ to $S_{\text {diss }}$ with one more water dissociated at the $\gamma-\mathrm{Al}_{2} \mathrm{O}_{3}(110) /$ water interface (highlighted in red in Figure 1). Such dissociation was not observed during the sampling of $\mathrm{FES}_{1}$ nor during a free molecular dynamics of the interface in absence of the ethanol. ${ }^{17}$ The adsorbed ethoxy may act as a pivot to allow hydrogen hopping between surface oxygens, enabling fast proton exchanges. After the desorption of ethanol, during the metadynamics run, no spontaneous return to the initial surface state $S_{r e f}$ was observed. As a consequence, the desorbed macro-state $\mu_{0} @ S_{\text {diss }}$ is not equivalent to the one obtained in $\mathrm{FES}_{1}\left(\mu_{0} @ S_{\text {ref }}\right)$. 
Hence, the comparison of the free energy of desorption obtained from $\mathrm{FES}_{1}$ and $\mathrm{FES}_{2}$ does not provide any information about the relative stability of $\mu_{1} @ S_{r e f}$ and $\mu_{2} @ S_{r e f}$ since they correspond to $\Delta F\left(\mu_{1} @ S_{r e f} \rightarrow \mu_{0} @ S_{r e f}\right)$ and $\Delta F\left(\mu_{2} @ S_{r e f} \rightarrow \mu_{0} @ S_{\text {diss }}\right)$ respectively. The impact of the change in surface state from $S_{r e f}$ to $S_{d i s s}$ will be investigated using complementary methods below.

Remarkably, the $\mu_{1, b i s}$ macro-state explored in $\mathrm{FES}_{2}$ differs from $\mu_{1} @ S_{r e f}$, which is used as a starting point to explore $\mathrm{FES}_{1}$. The position of the latter is highlighted in Figure 4 by a red circle. It is clearly not sampled during the simulation starting from $\mu_{2} @ S_{r e f}$ and hence is not an intermediate along the desorption from $\mu_{2} @ S_{r e f} \cdot \mu_{1, b i s}$ covers three different valleys with increasing solvation from 1 to 3 . At $\mathrm{CV}_{\text {solv }}=1$, this macro-state shows a shallow minimum. It corresponds to an ethoxy with a single $\mathrm{Al}-\mathrm{O}_{\text {ethanol }}$ bond. At a $\mathrm{CV}_{\text {solv }}=2$, the ethoxy is now protonated into ethanol and is loosing one $\mathrm{Al}-\mathrm{O}_{\text {ethanol }}$ bond. But, this bond scission is synchronous with the desorption process (increase in height, decrease in multiplicity from 1 to 0 ) with no identified minimum at this value of solvation in the $\mu_{1 b i s}$ zone. 


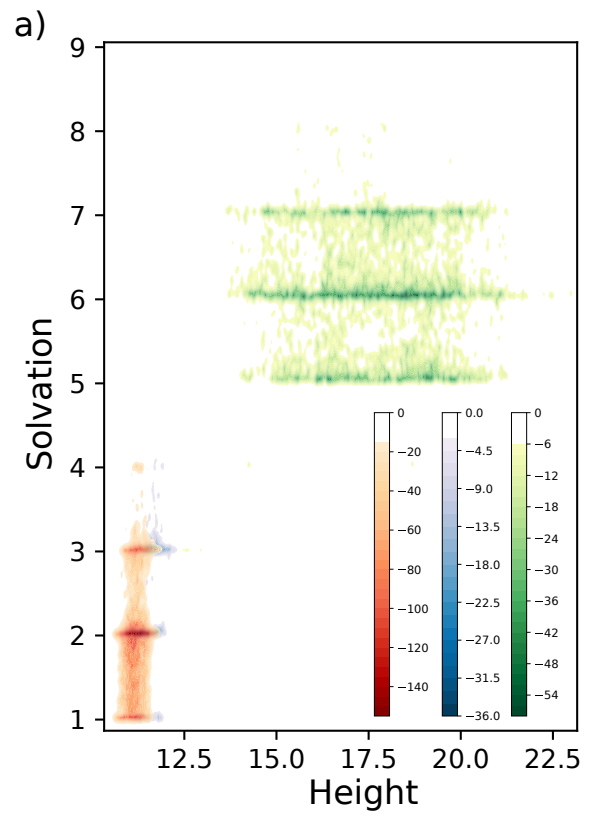

b)

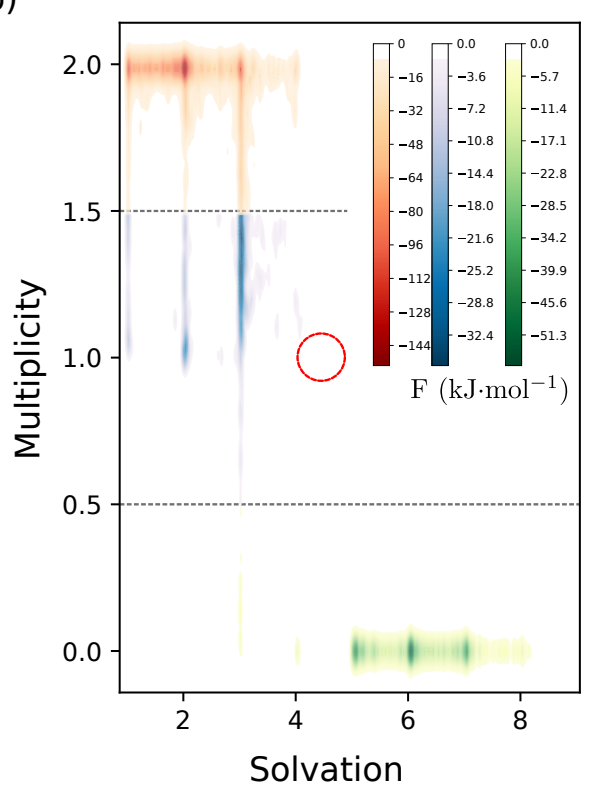

c)
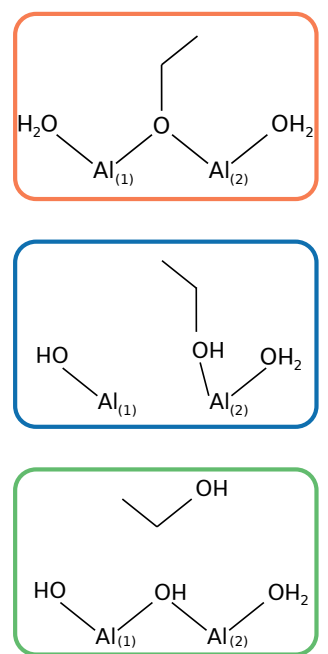

Figure 4: Free energy surface $F E S_{2}$ built using well-tempered metadynamics starting from $\mu_{2} @ S_{r e f}$, with the ethanol being chemisorbed dissociatively at the $\gamma-\mathrm{Al}_{2} \mathrm{O}_{3}(110) /$ water interface. (a) 2D-representation of the $3 \mathrm{D} F E S_{2}$ as a function of $\mathrm{CV}_{\text {solv }}$ and $\mathrm{CV}_{\text {height }}$ (b) 2D-representation of the 3DFES 2 as a function of $C V_{\text {multi }}$ and $\mathrm{CV}_{\text {solv }}$. (c) Schemes of a typical arrangement of $\mu_{2} @ S_{r e f}$ (orange), $\mu_{1 b i s}$ (blue) and $\mu_{0} @ S_{\text {diss }}$ (green). In (a) and (b), the $\mu_{2}$ macro-state $\left(\mathrm{CV}_{\text {multi }}>1.5\right.$, chemisorbed ethanol bridging two Al atoms) is shown using an orange scale, the $\mu_{1, \text { bis }}$ macro-state $\left(1.5>\mathrm{CV}_{\text {multi }}>0.5\right.$, with only one $\mathrm{Al}-\mathrm{O}_{\text {ethanol }}$ bond) using a blue scale and the $\mu_{0}$ macro-state $\left(\mathrm{CV}_{\text {multi }}<0.5\right.$, desorbed ethanol $)$ using a green scale. The boundaries are shown using grey dotted lines. The red circle stands for the approximate coordinates of $\mu_{1} @ S_{\text {ref }}$ using the CV used to build FES 2 . Note that the $\mathrm{CV}_{\text {solvation }}$ differs in $\mathrm{FES}_{1}$ and FES 2 (see section 2.4.1). Details about the reduction in dimension from 3D to 2D can be found in section 2.4.3. 


\subsection{Static approaches and classical solvation}

Benefiting from the mechanistic understanding provided by well-tempered metadynamics, the energetics of desorption were refined using static DFT calculations. Here, the alumina surface was explicitly hydrated by chemisorbed water molecules, some being dissociated. We considered the desorption from the $\mu_{1}$ and $\mu_{2}$ adsorption modes, combined with the two surface states $S_{r e f}$ and $S_{\text {diss }}$ of $\gamma-\mathrm{Al}_{2} \mathrm{O}_{3}(110)$ shown in Figure 1. Free energy differences were computed here based on the following substitution reactions:

$$
\begin{aligned}
& \mu_{1}-(\mathrm{EtOH}) @ \mathrm{Al}_{2} \mathrm{O}_{3}+\mathrm{H}_{2} \mathrm{O} \longrightarrow \mu_{0}-(\mathrm{EtOH})+\mathrm{H}_{2} \mathrm{O} @ \mathrm{Al}_{2} \mathrm{O}_{3} \\
& \mu_{2}-(\mathrm{EtO}) @ \mathrm{Al}_{2} \mathrm{O}_{3}+\mathrm{H}_{2} \mathrm{O} \longrightarrow \mu_{0}-(\mathrm{EtOH})+\mathrm{HO} @ \mathrm{Al}_{2} \mathrm{O}_{3}
\end{aligned}
$$

The results are presented in Figure 5 comparing three situations: (a) in absence of solvent (b) using a polarisable continuum model (PCM) of water solvent and (c) using a hybrid approach (MMsolv) to include explicitly the water solvent.

In absence of a solvent model (Figure 5 (a)), the reference surface state $S_{r e f}$ is $9 \mathrm{~kJ} \cdot \mathrm{mol}^{-1}$ less stable than the surface state $S_{\text {diss }}$ that presents an additional dissociated water molecule. This trend is maintained in presence of a $\mu_{2}-\mathrm{EtO}$ but is reversed in presence of a $\mu_{1}-\mathrm{EtOH}$ : $\mu_{1} @ S_{r e f}$ is $17 \mathrm{~kJ} \cdot \mathrm{mol}^{-1}$ more stable than $\mu_{1} @ S_{\text {diss }}$. However, since the $\mu_{1}$ adsorption is endergonic while the $\mu_{2}$ adsorption is exergonic, $S_{r e f}$ should never be exposed according to those results.

Upon the inclusion of water as a solvent using PCM, the relative stability of these surface states and adsorption modes is modified (Figure $5(\mathrm{~b})$ ). $S_{\text {ref }}$ is now found slightly more stable than $S_{\text {diss }}$ (by $5 \mathrm{~kJ} \cdot \mathrm{mol}^{-1}$ ), which is compatible with the surface model of alumina in water established by Réocreux and co-workers. ${ }^{17}$ The $\mu_{1}$ adsorption mode is found to be slightly exergonic on $S_{r e f}$, yielding to a stable $\mu_{1} @ S_{r e f}\left(\Delta \mathrm{G}_{a d s}=-7 \mathrm{~kJ} \cdot \mathrm{mol}^{-1}\right)$. But the most stable state remains $\mu_{2} @ S_{\text {diss, }}$ with $\Delta \mathrm{G}_{a d s}=-23 \mathrm{~kJ} \cdot \mathrm{mol}^{-1}$. This is in line 
with the $\mu_{2} @ S_{\text {diss }}$ state observed when exploring FES 2 and with the absence of $S_{\text {diss }}$ when exploring $\mathrm{FES}_{1}$.

Although implicit solvation is well-suited to describe indirect solvation effects like modifications in the long-range electrostatic interactions, it cannot provide a proper estimate of another significant contribution: the rearrangement of the hydrogen bonding network. In order to overcome this problem, we used MMSolv, ${ }^{25}$ an hybrid approach we recently developed to compute solvation free energy differences upon adsorption at a solid/liquid interface. ${ }^{25}$ This technique proceeds by freezing the surface and adsorbate in a given geometry and performing an extensive sampling of the water phase via molecular mechanics in order to determine the solvation difference between two states. MMSolv can therefore be considered as a static method, even though it relies on a dynamic sampling of the water phase, as it computes free energy difference between fixed conformations of the surface and adsorbates. The inclusion of explicit solvation contribution to the aforementioned free energies modifies the relative stability of the adsorption modes and surface state as depicted in Figure 5 (c). In absence of ethanol, $S_{\text {diss }}$ is destabilised by more than $25 \mathrm{~kJ} \cdot \mathrm{mol}^{-1}$ relative to $S_{\text {ref }}$. This is in line with the relative stability obtained using PCM. Besides, $\mu_{2} @ S_{\text {diss }}$ is still predicted to be the most RR: more? Il y en a que 2 il me semble $\rightarrow$ CM: Non, il y a aussi les mu1, ca fait 4 tout. stable adsorption mode of ethanol $\left(-28 \mathrm{~kJ} \cdot \mathrm{mol}^{-1}\right)$, in agreement with the gas phase $\left(-19 \mathrm{~kJ} \cdot \mathrm{mol}^{-1}\right)$ and PCM $\left(-23 \mathrm{~kJ} \cdot \mathrm{mol}^{-1}\right)$. Noticeably, the difference with the second most stable adsorption mode $\left(\mu_{1} @ S_{r e f}\right)$ drops below $10 \mathrm{~kJ} \cdot \mathrm{mol}^{-1}$ upon the explicit description of the water solvent. Explicit solvation has a greater impact on $\mu_{1}$ than on $\mu_{2}$ since $\mu_{1}$ (only one $\mathrm{Al}-\mathrm{O}_{\mathrm{EtOH}}$ bond) can more easily build $\mathrm{H}$-bond with water than $\mu_{2}$ (two $\mathrm{Al}-\mathrm{O}_{\mathrm{EtOH}}$ bonds). 


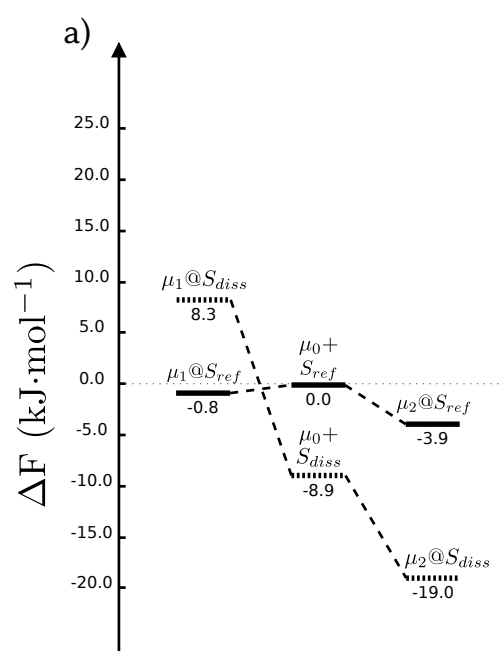

Gas phase

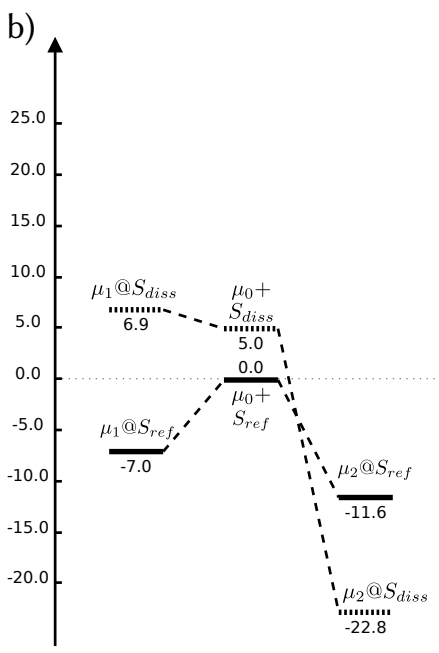

Implicit solvation

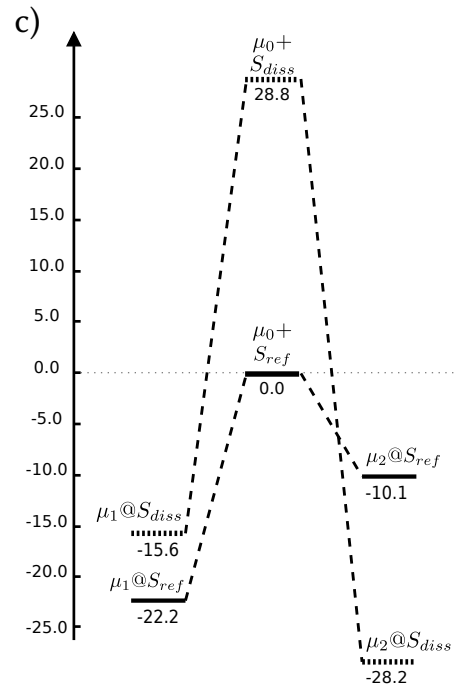

MMSolv

Figure 5: Free energy diagram of the adsorption of ethanol at the $\gamma-\mathrm{Al}_{2} \mathrm{O}_{3}(110) /$ water interface considering two models of the fully hydrated surface $S_{r e f}$ and $S_{d i s s}$ and two adsorption modes $\mu_{1}$ and $\mu_{2}$. In (a), the bulk water solvent is neglected while it is included in (b) by using a polarisable continuum model and in (c) using the hybrid scheme MMSolv. ${ }^{25}$ All free energies are given in $\mathrm{kJ} \cdot \mathrm{mol}^{-1}$ relatively to $S_{\text {ref }}$. 


\subsection{Thermodynamic Integration}

Thermodynamic integration (TI) was used to refine the energetics of the desorption of ethanol with a better sampling and an improved accuracy. The free energy profiles along the height $\left(\mathrm{CV}_{\text {height }}\right.$, using the same definition as in the well-tempered metadynamics, see 2.4.1) were computed starting from $\mu_{1} @ S_{r e f}, \mu_{2} @ S_{r e f}$ but also $\mu_{2} @ S_{\text {diss }}$.

As presented in Figure 6, the three desorption profiles determined by TI present a transition state around $12.5 \AA$, close to the one observed in the free energy surfaces FES 1 and $\mathrm{FES}_{2}$ that were reconstructed using well-tempered metadynamics (see Figures 3 and 4). At this height, the ethanol molecule lies in a partially structured water identified previously as a physisorbed layer. ${ }^{17}$ The tails of the different profiles were merged above a certain height threshold (see computational details) since the three systems become chemically equivalent $\left(\mu_{0} @ S_{r e f}\right)$.

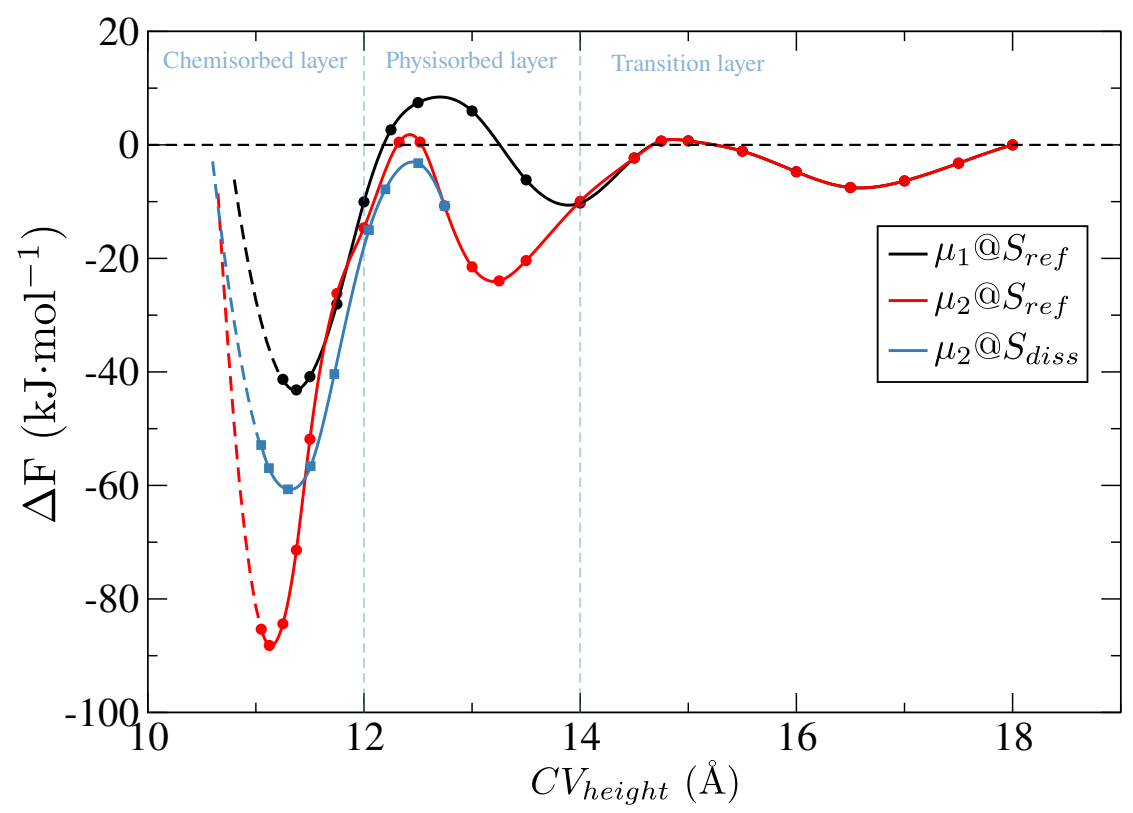

Figure 6: Free energy profiles $\Delta F$ as a function of $C V_{\text {height }}$ computed with thermodynamic integration for the desorption from $\mu_{2} @ S_{d i s s}, \mu_{2} @ S_{r e f}$, and $\mu_{1} @ S_{r e f}$. The dashed parts of the profiles represent an extrapolation at small displacement along $C V_{\text {height }}$ determined by a quadratic fit. The profiles are merged for $C V_{\text {height }}>14 \AA$ as explained in the computational details. The light blue vertical lines represent the limit of the structured water layers of water evidenced by Réocreux et al. ${ }^{17}$ 
Desorption free energies and the associated activation barriers were determined applying Equations 4 and 5 to the TI free energy profiles. The corresponding data are reported in Figure 7.

The desorption free energy from $\mu_{1} @ S_{r e f}$ is endothermic according to the TI (+31 $\mathrm{kJ} \cdot \mathrm{mol}^{-1}$ ) while it was athermic to exothermic according to the well-tempered metadynamics $\left(-9 \mathrm{~kJ} \cdot \mathrm{mol}^{-1}\right)$. Despite a careful and extended sampling in the TI, the free energy gradients do not strictly tend towards zero at long distances (and the free energy does not converge to a constant) as should be observed when ethanol approaches the bulkwater zone. This behaviour indicates that the sampling of the $\mu_{0} @ S_{r e f}$ macro-state is here again not exhaustive, the CPU cost required to sample all slow degrees of freedom of the desorbed ethanol (diffusion in the in-plane direction, rotations of the alkyl chain) and to sample the reorganisation of the solvation shells (proton transfers and water diffusion) being non affordable. This incomplete sampling of the $\mu_{0} @ S_{r e f}$ macro-state is likely at the origin of these differences in desorption free energies between the TI and the well-tempered metadynamics. Noticeably, it appears that the desorption of ethanol from $\mu_{1} @ S_{r e f}$ is also found to be endothermic using static approaches in a 7 to $22 \mathrm{~kJ} \cdot \mathrm{mol}^{-1}$ range, depending on the solvent model (see Figure 5). The desorption barrier from $\mu_{1} @ S_{r e f}$ differs also between the TI $\left(75 \mathrm{~kJ} \cdot \mathrm{mol}^{-1}\right)$ and the metadynamics $\left(35 \mathrm{~kJ} \cdot \mathrm{mol}^{-1}\right)$. Since the height is used as a unique $\mathrm{CV}$ in the $\mathrm{TI}$, variations in solvation is likely to be missing in the TI. It was necessary, after the desorption of $\mathrm{EtOH}$, to run a preliminary slow-growth simulation to pull a water molecule towards the $\mathrm{Al}$ adsorption site in order to shorten the equilibration time and, therefore, possibly to cross the approximately $30 \mathrm{~kJ} \cdot \mathrm{mol}^{-1}$ barrier evidenced with metadynamics to increase the solvation variable from 3 to 4 prior desorption. This hidden variable in the TI may be responsible for the observed discrepancy between TI and $\mathrm{FES}_{1}$ obtained by well-tempered metadynamics.

Regarding the bidentate ethoxy adsorption $\mu_{2}$, the mechanistic observations made during the sampling of $\mathrm{FES}_{2}$ lead to consider here two configurations: $\mu_{2} @ S_{r e f}$ and $\mu_{2} @ S_{\text {diss }}$. 
In both cases, the $\mu_{0} @ S_{r e f}$ is obtained as a final state of desorption. This common final desorption macro-state $\mu_{0} @ S_{r e f}$ contrasts with the $\mu_{0} @ S_{\text {diss }}$ macro-state obtained during the well-tempered metadynamics starting from $\mu_{2} @ S_{r e f}$. For the TI starting from $\mu_{2} @ S_{r e f}$, the surface state is not modified while for the TI starting from $\mu_{2} @ S_{\text {diss }}$, the $S_{\text {diss }}$ surface spontaneously reconstructed into $S_{\text {ref }}$ for $C V_{\text {height }}>12.5 \AA$. The proton-shuffling was not biased in the TI and is limited by the unavoidably too-short molecular dynamics runs while the solvation variable triggers the proton exchanges between the ethoxy and all water molecules during the sampling of $\mathrm{FES}_{2}$. Still, reaching a common final macro-state $\mu_{0} @ S_{r e f}$ when using TI allows us to compare the stability of those two adsorption modes: the $\mu_{2} @ S_{r e f}$ is found to be more stable than $\mu_{2} @ S_{\text {diss }}$ by $32 \mathrm{~kJ} \cdot \mathrm{mol}^{-1}$. This appears in contradiction with the static approaches that systematically identified $\mu_{2} @ S_{d i s s}$ as the most stable $\mu_{2}$ adsorption mode (Figure 5) by at least $10 \mathrm{~kJ} \cdot \mathrm{mol}^{-1}$. This is likely related to the level of description of the water as a solvent and illustrate the necessity of an improved description of water/oxide interfaces. The barrier of desorption from $\mu_{2} @ S_{r e f}$ is found to be of $89 \mathrm{~kJ} \cdot \mathrm{mol}^{-1}$, which is clearly lower than the one found of the $\mathrm{FES}_{2}\left(149 \mathrm{~kJ} \cdot \mathrm{mol}^{-1}\right)$. Here, hidden variables as solvation may also be at the origin of the underestimation when using TI compared with well-tempered metadynamics. Still, those two approaches provide consistent mechanistic information when considering the evolution of the number of hydrogen bonds between $\mathrm{EtOH}$ and water or of the number of $\mathrm{Al}-\mathrm{O}$ bonds upon desorption (see Figures S2 and S3). Prior desorption $\left(C V_{\text {height }}<12.5 \AA\right)$, the ethoxy is protonated while the multiplicity drops from 2 to 0 with no marked minimum corresponding to $\mu_{1}$. After desorption, the solvation increases through the hydration of the two aluminium atoms, with two additional $\mathrm{Al}-\mathrm{O}_{\text {water }}$ bonds, and through the hydration of ethanol, building 1 or 2 hydrogen bonds with water. 


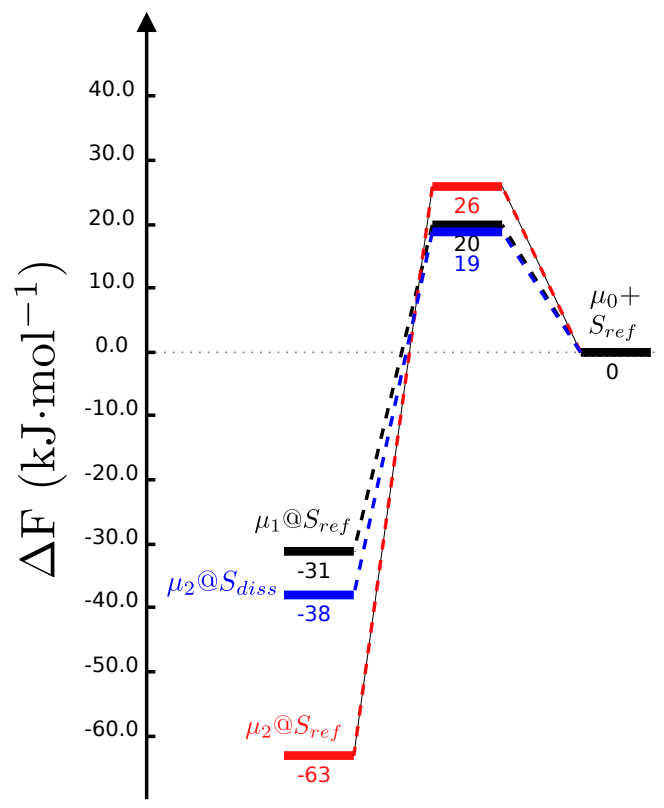

Figure 7: Free energy levels $\Delta F$ computed with thermodynamic integration for the desorption from $\mu_{2} @ S_{\text {diss }}, \mu_{2} @ S_{r e f}$, and $\mu_{1} @ S_{r e f}$. All free energy levels are determined by the weighted average method described in computational details (Equations 4 and 5) with $C V_{\text {heigt }}=12.5 \AA$ taken as boundary between the two region of the curve (adsorbed for $C V_{\text {heigt }}<12.5 \AA$, desorbed for $C V_{\text {heigt }}>12.5 \AA$ ). Figures are given within a $3 \mathrm{~kJ} \cdot \mathrm{mol}^{-1}$ uncertainty. 


\section{Conclusion}

The desorption of ethanol from the $\gamma$-alumina/water interface to the bulk water was investigated combining several computational methods sharing the same level of theory to evaluate energy (using PBE+D3 as a DFT functional). Using well-tempered ab initio metadynamics, two free energy surfaces were reconstructed starting from two possible adsorption modes of ethanol: (i) ethanol is kept intact and it interacts with only one aluminium atom $\left(\mu_{1}\right)$, (ii) ethanol is dissociated and the corresponding ethoxy bridges two aluminium atoms $\left(\mu_{2}\right)$. Those metadynamic simulations highlight the important role of solvation of both ethanol and alumina during the desorption process. Increasing solvation of ethanol is necessary to open the desorption route and happens prior desorption. On the other hand, the alumina surface is hydrated later, concomitantly to the translation of ethanol above $2.5 \AA$ away from the alumina surface. When starting from the chemisorbed ethoxy bridging two aluminium atoms, its protonation precedes the desorption, but the monodentate ethanol does not appears as a minimum along the desorption pathway. In addition, we observe a change in the surface state during the desorption from $\mu_{2}$, from the

reference surface state $S_{r e f}$ to a surface with an extra dissociated chemisorbed water $S_{\text {diss }}$. This is likely to be triggered by the bias along our set of collective variables that includes solvation.

The impact of the surface state on the adsorption of ethanol was further investigated using static methods combined with two solvent models. They consistently found the ethoxy adsorption mode $\mu_{2}$ on $S_{\text {diss }}$ being the most stable adsorption of ethanol at the $\gamma$-alumina/water interface while $S_{\text {diss }}$ is less stable than $S_{r e f}$ in absence of a chemisorbed ethanol.

To refine the energetic of desorption, we performed thermodynamic integration starting from three adsorption configurations, namely $\mu_{1} @ S_{r e f}, \mu_{2} @ S_{r e f}$, and $\left.\mu_{2} @ S_{d i s s}\right)$. With only one variable, this is supposedly easier to converge than a 3D-FES using ab initio welltempered metadynamics. Still, in absence of a bias to trigger the changes in solvation 
shells, we had to manually bring a water molecule closer to the vacant surface site to hydrate the surface. This 'hidden variable' may explain the discrepancies observed in the energetic of desorption between well-tempered metadyanmics and thermodynamic integration. Still, they both identified $\mu_{2}$ as the most stable adsorption mode, in agreement with static methods. The desorption energy of $\mu_{2}$ is found ranging 60 to $90 \mathrm{~kJ} \cdot \mathrm{mol}^{-1}$ which is stronger than in absence of any solvent $\left(19 \mathrm{~kJ} \cdot \mathrm{mol}^{-1}\right)$. The changes in solvation are responsible for a desorption barrier evaluated around 90 to $149 \mathrm{~kJ} \cdot \mathrm{mol}^{-1}$ depending on the method. This cannot be easily determined using cheaper methods and the differences observed between well-tempered metadynamics and thermodynamic integration calls for the development of better approaches to investigate the reactive desorption/adsorption at the solid/water interface.

\section{Acknowledgement}

This work is part of the "RatiOnAl Design for CATalysis" (ROAD4CAT) industrial chair, project IDEXLYON funded by the French National Research Agency (ANR-16-IDEX-0005) and the Commissariat-General for Investment (CGI) within the framework of Investissements d'Avenir program ("Investment for the future"). The authors thank the SYSPROD project and AXELERA Pôle de Compétitivité for financial support (PSMN Data Center). This work was also granted access to the HPC resources of CINES and IDRIS under the allocation 0800609 made by GENCI. We thank Pascal Raybaud for his insightful discussions.

\section{Supporting Information Available}

Figures showing the decomposition analysis of the collective variables evolution. Thermodynamic intergrations data (cumulative error, free energy gradient, the standard error on 
the mean values of the fre energy gradient). A representative structure of $\mu_{1} @ S_{r e f}, \mu_{2} @ S_{r e f}$, $\mu_{2} @ S_{\text {diss }}$ in .xyz format. The HILLS files from the two metadynamics. Typical input files of a TI and for each well-tempered metadynamics.

\section{References}

(1) Shenghua, L.; He, Y.; Yuansheng, J. Lubrication Chemistry Viewed from DFT-Based Concepts and Electronic Structural Principles. Int. J. Mol. Sci. 2003, 5, 13.

(2) Pirkanniemi, K.; Sillanpää, M. Heterogeneous water phase catalysis as an environmental application: a review. Chemosphere 2002, 48, 1047-1060.

(3) Akpa, B. S.; D'Agostino, C.; Gladden, L. F.; Hindle, K.; Manyar, H.; McGregor, J.; Li, R.; Neurock, M.; Sinha, N.; Stitt, E. H. et al. Solvent effects in the hydrogenation of 2-butanone. J. Catal. 2012, 289, 30.

(4) Seh, Z. W.; Kibsgaard, J.; Dickens, C. F.; Chorkendorff, I.; Nørskov, J. K.; Jaramillo, T. F. Combining theory and experiment in electrocatalysis: Insights into materials design. Science 2017, 355, eaad4998.

(5) Verdaguer, A.; Sacha, G. M.; Bluhm, H.; Salmeron, M. Molecular Structure of Water at Interfaces: Wetting at the Nanometer Scale. Chemical Reviews 2006, 106, 1478-1510, Publisher: American Chemical Society.

(6) Maier, S.; Salmeron, M. How Does Water Wet a Surface? Accounts of Chemical Research 2015, 48, 2783-2790, Publisher: American Chemical Society.

(7) Björneholm, O.; Hansen, M. H.; Hodgson, A.; Liu, L.-M.; Limmer, D. T.; Michaelides, A.; Pedevilla, P.; Rossmeisl, J.; Shen, H.; Tocci, G. et al. Water at Interfaces. Chem. Rev. 2016, 116, 7698-7726. 
(8) Schiros, T.; Andersson, K. J.; Pettersson, L. G. M.; Nilsson, A.; Ogasawara, H. Chemical bonding of water to metal surfaces studied with core-level spectroscopies. Journal of Electron Spectroscopy and Related Phenomena 2010, 177, 85-98.

(9) Velasco-Velez, J.-J.; Wu, C. H.; Pascal, T. A.; Wan, L. F.; Guo, J.; Prendergast, D.; Salmeron, M. The structure of interfacial water on gold electrodes studied by $\mathrm{x}$-ray absorption spectroscopy. Science 2014, 346, 831-834.

(10) Toney, M. F.; Howard, J. N.; Richer, J.; Borges, G. L.; Gordon, J. G.; Melroy, O. R.; Wiesler, D. G.; Yee, D.; Sorensen, L. B. Voltage-dependent ordering of water molecules at an electrode-electrolyte interface. Nature 1994, 368, 444-446.

(11) Michel, C.; Gallezot, P. Why Is Ruthenium an Efficient Catalyst for the AqueousPhase Hydrogenation of Biosourced Carbonyl Compounds? ACS Catalysis 2015, 5, 4130-4132.

(12) Calvin, J. J.; Rosen, P. F.; Ross, N. L.; Navrotsky, A.; Woodfield, B. F. Review of surface water interactions with metal oxide nanoparticles. Journal of Materials Research 2019, 34, 416-427, WOS:000458762500007.

(13) Foucaud, Y.; Badawi, M.; Filippov, L. O.; Filippova, I. V.; Lebègue, S. Surface Properties of Fluorite in Presence of Water: An Atomistic Investigation. The Journal of Physical Chemistry B 2018, 122, 6829-6836.

(14) Walsh, T. R. Pathways to Structure-Property Relationships of Peptide-Materials Interfaces: Challenges in Predicting Molecular Structures. Accounts of Chemical Research 2017, 50, 1617-1624, WOS:000406085500013.

(15) Leung, K.; Criscenti, L. J.; Knight, A. W.; Ilgen, A. G.; Ho, T. A.; Greathouse, J. A. Concerted Metal Cation Desorption and Proton Transfer on Deprotonated Silica Surfaces. The Journal of Physical Chemistry Letters 2018, 9, 5379-5385. 
(16) Saleheen, M.; Heyden, A. Liquid-Phase Modeling in Heterogeneous Catalysis. ACS Catalysis 2018, 8, 2188-2194.

(17) Réocreux, R.; Jiang, T.; Iannuzzi, M.; Michel, C.; Sautet, P. Structuration and Dynamics of Interfacial Liquid Water at Hydrated $\gamma$-Alumina Determined by ab Initio Molecular Simulations: Implications for Nanoparticle Stability. ACS Appl. Nano Mater. 2018, 1, 191-199.

(18) Wright, L. B.; Walsh, T. R. Facet Selectivity of Binding on Quartz Surfaces: Free Energy Calculations of Amino-Acid Analogue Adsorption. Journal of Physical Chemistry C 2012, 116, 2933-2945, WOS:000300462400035.

(19) Ali, A.; Le, T. T. B.; Striolo, A.; Cole, D. R. Salt Effects on the Structure and Dynamics of Interfacial Water on Calcite Probed by Equilibrium Molecular Dynamics Simulations. The Journal of Physical Chemistry C 2020, 124, 24822-24836, Publisher: American Chemical Society.

(20) Faheem, M.; Heyden, A. Hybrid Quantum Mechanics/Molecular Mechanics Solvation Scheme for Computing Free Energies of Reactions at Metal-Water Interfaces. J. Chem. Theory Comput. 2014, 10, 3354-3368.

(21) Steinmann, S. N.; Sautet, P.; Michel, C. Solvation free energies for periodic surfaces: comparison of implicit and explicit solvation models. Physical Chemistry Chemical Physics 2016, 18, 31850-31861.

(22) Lim, H.-K.; Lee, H.; Kim, H. A Seamless Grid-Based Interface for Mean-Field QM/MM Coupled with Efficient Solvation Free Energy Calculations. J. Chem. Theory Comput. 2016, 12, 5088-5099, Publisher: American Chemical Society.

(23) Naserifar, S.; Chen, Y.; Kwon, S.; Xiao, H.; Goddard, W. A. Artificial Intelligence and QM/MM with a Polarizable Reactive Force Field for Next-Generation Electrocatalysts. Matter 2021, 4, 195-216. 
(24) Zhang, X.; DeFever, R. S.; Sarupria, S.; Getman, R. B. Free Energies of Catalytic Species Adsorbed to Pt(111) Surfaces under Liquid Solvent Calculated Using Classical and Quantum Approaches. Journal of Chemical Information and Modeling 2019,

(25) Clabaut, P.; Schweitzer, B.; Götz, A. W.; Michel, C.; Steinmann, S. N. Solvation Free Energies and Adsorption Energies at the Metal/Water Interface from Hybrid QuantumMechanical/Molecular Mechanics Simulations. J. Chem. Theory Comput. 2020, 16, 6539-6549.

(26) Li, C.; Monti, S.; Carravetta, V. Journey toward the Surface: How Glycine Adsorbs on Titania in Water Solution. Journal of Physical Chemistry C 2012, 116, 18318-18326, WOS:000308120000040.

(27) Raju, M.; Kim, S.-Y.; van Duin, A. C. T.; Fichthorn, K. A. ReaxFF Reactive Force Field Study of the Dissociation of Water on Titania Surfaces. Journal of Physical Chemistry C 2013, 117, 10558-10572, WOS:000319649400037.

(28) Hahn, S. H.; van Duin, A. C. T. Surface Reactivity and Leaching of a Sodium Silicate Glass under an Aqueous Environment: A ReaxFF Molecular Dynamics Study. The Journal of Physical Chemistry C 2019, 123, 15606-15617.

(29) Zhao, Y. L.; Köppen, S.; Frauenheim, T. An SCC-DFTB/MD Study of the Adsorption of Zwitterionic Glycine on a Geminal Hydroxylated Silica Surface in an Explicit Water Environment. The Journal of Physical Chemistry C 2011, 115, 9615-9621.

(30) Spiegelman, F.; Tarrat, N.; Cuny, J.; Dontot, L.; Posenitskiy, E.; Martí, C.; Simon, A.; Rapacioli, M. Density-functional tight-binding: basic concepts and applications to molecules and clusters. Advances in Physics: X 2020, 5, 1710252.

(31) Artrith, N.; Kolpak, A. M. Understanding the Composition and Activity of Electrocatalytic Nanoalloys in Aqueous Solvents: A Combination of DFT and Accurate Neural Network Potentials. Nano Letters 2014, 14, 2670-2676. 
(32) Behler, J. Constructing high-dimensional neural network potentials: A tutorial review. International Journal of Quantum Chemistry 2015, 115, 1032-1050.

(33) Natarajan, S. K.; Behler, J. Neural network molecular dynamics simulations of solid-liquid interfaces: water at low-index copper surfaces. Physical Chemistry Chemical Physics 2016, 18, 28704-28725.

(34) Laio, A.; Parrinello, M. Escaping free-energy minima. PNAS 2002, 99, 12562-12566, Publisher: National Academy of Sciences Section: Physical Sciences.

(35) Laio, A.; Gervasio, F. L. Metadynamics: a method to simulate rare events and reconstruct the free energy in biophysics, chemistry and material science. Rep. Prog. Phys. $2008,23$.

(36) Liu, L.-M.; Laio, A.; Michaelides, A. Initial stages of salt crystal dissolution determined with ab initio molecular dynamics. Phys. Chem. Chem. Phys. 2011, 13, 13162.

(37) Schneider, J.; Colombi Ciacchi, L. Specific Material Recognition by Small Peptides Mediated by the Interfacial Solvent Structure. J. Am. Chem. Soc. 2012, 134, 2407-2413.

(38) Käner, J. Umbrella sampling. WIREs Computational Molecular Science 2011, 1, 932-942.

(39) Tazi, S.; Rotenberg, B.; Salanne, M.; Sprik, M.; Sulpizi, M. Absolute acidity of clay edge sites from ab-initio simulations. Geochimica et Cosmochimica Acta 2012, 94, 1-11.

(40) Parashar, S.; Lesnicki, D.; Sulpizi, M. Increased Acid Dissociation at the Quartz/Water Interface. J. Phys. Chem. Lett. 2018, 9, 2186-2189.

(41) Bailleul, S.; Rogge, S. M. J.; Vanduyfhuys, L.; Van Speybroeck, V. Insight into the Role of Water on the Methylation of Hexamethylbenzene in H-SAPO-34 from First Principle Molecular Dynamics Simulations. Chem CatChem 2019, 11, 3993-4010.

(42) Michaelis, M.; Delle Piane, M.; Rothenstein, D.; Perry, C. C.; Colombi Ciacchi, L. Lessons from a Challenging System: Accurate Adsorption Free Energies at the Amino 
Acid/ZnO Interface. Journal of Chemical Theory and Computation 0, 0, null, PMID: 34191508 .

(43) Réocreux, R.; Girel, E.; Clabaut, P.; Tuel, A.; Besson, M.; Chaumonnot, A.; Cabiac, A.; Sautet, P.; Michel, C. Reactivity of shape-controlled crystals and metadynamics simulations locate the weak spots of alumina in water. Nature Communications 2019, 10.

(44) Copeland, J. R.; Shi, X.-R.; Sholl, D. S.; Sievers, C. Surface Interactions of $C_{2}$ and $\mathrm{C}_{3}$ Polyols with $\gamma-\mathrm{Al}_{2} \mathrm{O}_{3}$ and the Role of Coadsorbed Water. Langmuir 2013, 29, 581-593.

(45) Copeland, J. R.; Santillan, I. A.; Schimming, S. M.; Ewbank, J. L.; Sievers, C. Surface Interactions of Glycerol with Acidic and Basic Metal Oxides. J. Phys. Chem. C 2013, 117, 21413-21425, Publisher: American Chemical Society.

(46) Krokidis, X.; Raybaud, P.; Gobichon, A. E.; Rebours, B.; Euzen, P.; Toulhoat, H. Theoretical study of the dehydration process of boehmite to gamma-alumina. Journal of Physical Chemistry B 2001, 105, 5121-5130, WOS:000169232800008.

(47) Digne, M.; Sautet, P.; Raybaud, P.; Euzen, P.; Toulhoat, H. Hydroxyl Groups on $\gamma$-Alumina Surfaces: A DFT Study. Journal of Catalysis 2002, 211, 1-5.

(48) Wischert, R.; Laurent, P.; Coperet, C.; Delbecq, F.; Sautet, P. gamma-Alumina: The Essential and Unexpected Role of Water for the Structure, Stability, and Reactivity of "Defect" Sites. Journal of the American Chemical Society 2012, 134, 14430-14449, WOS:000308283200031.

(49) VandeVondele, J.; Krack, M.; Mohamed, F.; Parrinello, M.; Chassaing, T.; Hutter, J. QUICKSTEP: Fast and accurate density functional calculations using a mixed Gaussian and plane waves approach. Computer Physics Communications 2005, 167, 103-128, WOS:000228421500005. 
(50) VandeVondele, J.; Hutter, J. Gaussian basis sets for accurate calculations on molecular systems in gas and condensed phases. Journal of Chemical Physics 2007, 127, 114105, WOS:000249667400011.

(51) Lippert, G.; Hutter, J.; Parrinello, M. A hybrid Gaussian and plane wave density functional scheme. Molecular Physics 1997, 92, 477-487, WOS:A1997YC60700017.

(52) Hutter, J.; Iannuzzi, M.; Schiffmann, F.; VandeVondele, J. CP2K: atomistic simulations of condensed matter systems. Wiley Interdisciplinary Reviews: Computational Molecular Science 2014, 4, 15-25.

(53) Goedecker, S.; Teter, M.; Hutter, J. Separable dual-space Gaussian pseudopotentials. Physical Review B 1996, 54, 1703-1710, WOS:A1996UZ86100053.

(54) Hartwigsen, C.; Goedecker, S.; Hutter, J. Relativistic separable dual-space Gaussian pseudopotentials from H to Rn. Physical Review B 1998, 58, 3641-3662, WOS:000075616800043.

(55) Krack, M. Pseudopotentials for $\mathrm{H}$ to $\mathrm{Kr}$ optimized for gradient-corrected exchange-correlation functionals. Theoretical Chemistry Accounts 2005, 114, 145-152, WOS:000232053800020.

(56) Perdew, J. P.; Burke, K.; Ernzerhof, M. Generalized Gradient Approximation Made Simple. Physical Review Letters 1996, 77, 3865-3868.

(57) Grimme, S.; Antony, J.; Ehrlich, S.; Krieg, H. A consistent and accurate ab initio parametrization of density functional dispersion correction (DFT-D) for the 94 elements H-Pu. The Journal of Chemical Physics 2010, 132, 154104.

(58) Bussi, G.; Donadio, D.; Parrinello, M. Canonical sampling through velocity rescaling. Journal of Chemical Physics 2007, 126, 014101, WOS:000243380000005. 
(59) Tuckerman, M. E. Statistical Mechanics: Theory and Molecular Simulation; Oxford University Press, 2010.

(60) Barducci, A.; Bussi, G.; Parrinello, M. Well-Tempered Metadynamics: A Smoothly Converging and Tunable Free-Energy Method. Physical Review Letters 2008, 100.

(61) Tribello, G. A.; Bonomi, M.; Branduardi, D.; Camilloni, C.; Bussi, G. PLUMED 2: New feathers for an old bird. Computer Physics Communications 2014, 185, 604-613, WOS:000329537500020.

(62) Kresse, G. Ab initio molecular dynamics for liquid metals. Journal of Non-Crystalline Solids 1995, 192-193, 222-229.

(63) Kresse, G.; Furthmüller, J. Efficient iterative schemes for ab initio total-energy calculations using a plane-wave basis set. Physical Review B 1996, 54, 11169-11186.

(64) Perdew, J. P.; Wang, Y. Accurate and simple analytic representation of the electron-gas correlation energy. Phys. Rev. B 1992, 45, 13244-13249.

(65) Steinmann, S. N.; Corminboeuf, C. Comprehensive Benchmarking of a DensityDependent Dispersion Correction. J. Chem. Theory Comput. 2011, 7, 3567-3577.

(66) Gautier, S.; Steinmann, S. N.; Michel, C.; Fleurat-Lessard, P.; Sautet, P. Molecular adsorption at $\mathrm{Pt}(111)$. How accurate are DFT functionals? Phys. Chem. Chem. Phys. 2015, 17, 28921.

(67) Blochl, P. E. Projector augmented-wave method. 27.

(68) Kresse, G.; Joubert, D. From ultrasoft pseudopotentials to the projector augmentedwave method. Physical Review B 1999, 59, 1758-1775.

(69) Mathew, K.; Sundararaman, R.; Letchworth-Weaver, K.; Arias, T. A.; Hennig, R. G. Implicit solvation model for density-functional study of nanocrystal surfaces and reaction pathways. The Journal of Chemical Physics 2014, 140, 084106. 
(70) Wertz, D. H. Relationship between the gas-phase entropies of molecules and their entropies of solvation in water and 1-octanol. J. Am. Chem. Soc. 1980, 102, 5316-5322.

(71) Skelton, A. A.; Fenter, P.; Kubicki, J. D.; Wesolowski, D. J.; Cummings, P. T. Simulations of the Quartz(1011)/Water Interface: A Comparison of Classical Force Fields, Ab Initio Molecular Dynamics, and X-ray Reflectivity Experiments. J. Phys. Chem. C 2011, $115,2076-2088$.

(72) Rey, J.; Blanck, S.; Clabaut, P.; Loehlé, S.; Steinmann, S.; Michel, C. Transferable Gaussian Attractive Potentials for Organic/oxide Interfaces. 2021, Publisher: ChemRxiv.

(73) Rappe, A. K.; Casewit, C. J.; Colwell, K. S.; Goddard, W. A.; Skiff, W. M. UFF, a full periodic table force field for molecular mechanics and molecular dynamics simulations. Journal of the American Chemical Society 1992, 114, 10024-10035. 


\section{Graphical TOC Entry}

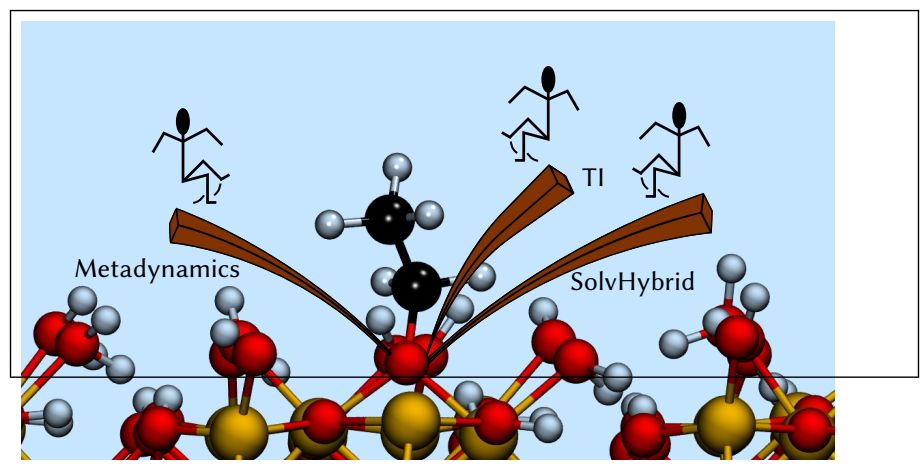

